\title{
SPECIALIZATIONS OF MULTIGRADINGS AND THE ARITHMETICAL RANK OF LATTICE IDEALS
}

\author{
ANARGYROS KATSABEKIS AND APOSTOLOS THOMA
}

\begin{abstract}
In this article we study specializations of multigradings and apply them to the problem of the computation of the arithmetical rank of a lattice ideal $I_{L_{\mathcal{G}}} \subset K\left[x_{1}, \ldots, x_{n}\right]$. The arithmetical rank of $I_{L_{\mathcal{G}}}$ equals the $\mathcal{F}$-homogeneous arithmetical rank of $I_{L_{\mathcal{G}}}$, for an appropriate specialization $\mathcal{F}$ of $\mathcal{G}$. To the lattice ideal $I_{L_{\mathcal{G}}}$ and every specialization $\mathcal{F}$ of $\mathcal{G}$ we associate a simplicial complex. We prove that combinatorial invariants of the simplicial complex provide lower bounds for the $\mathcal{F}$ homogeneous arithmetical rank of $I_{L_{\mathcal{G}}}$.
\end{abstract}

\section{INTRODUCTION}

On the polynomial ring $S=K\left[x_{1}, \ldots, x_{n}\right]$ with coefficients in a field $K$ one can impose several multigradings defined by abelian groups. Let $\mathcal{G}$ be a finitely generated abelian group together with a distinguished ordered set $\left\{\mathbf{g}_{1}, \ldots, \mathbf{g}_{n}\right\}$ of $n$ generators. The degree map

$$
\operatorname{deg}_{\mathcal{G}}: \mathbb{Z}^{n} \rightarrow \mathcal{G}, \operatorname{deg}_{\mathcal{G}}(\mathbf{u})=u_{1} \mathbf{g}_{1}+\cdots+u_{n} \mathbf{g}_{n} \text { for } \mathbf{u}=\left(u_{1}, \ldots, u_{n}\right) \in \mathbb{Z}^{n},
$$

defines a multigrading on $S$ by $\mathcal{G}$. The $\mathcal{G}$-degree of the monomial $x_{1}^{u_{1}} \cdots x_{n}^{u_{n}}$ is $\operatorname{deg}_{\mathcal{G}}(\mathbf{u})$. A polynomial $F \in S$ is called $\mathcal{G}$-homogeneous if the monomials in each non zero term of $F$ have the same $\mathcal{G}$-degree. An ideal $J$ is called $\mathcal{G}$-homogeneous if it is generated by $\mathcal{G}$-homogeneous polynomials.

The grading on $S$ by $\mathcal{G}$ defines the exact sequence

$$
0 \longrightarrow L_{\mathcal{G}} \stackrel{i}{\longrightarrow} \mathbb{Z}^{n} \stackrel{\operatorname{deg}_{\mathcal{G}}}{\longrightarrow} \mathcal{G} \longrightarrow 0 .
$$

Depending on the emphasis given to the group $\mathcal{G}$ or the lattice $L_{\mathcal{G}}$ it is called $\mathcal{G}$-grading or $L_{\mathcal{G}}$-grading. Remark that $\mathcal{G}$ together with the set $\left\{\mathbf{g}_{1}, \ldots, \mathbf{g}_{n}\right\}$ determines the lattice

$$
L_{\mathcal{G}}=\left\{\mathbf{u} \in \mathbb{Z}^{n}: \operatorname{deg}_{\mathcal{G}}(\mathbf{u})=\mathbf{0}_{\mathcal{G}}\right\} \subset \mathbb{Z}^{n}
$$

of relations of $\mathbf{g}_{1}, \ldots, \mathbf{g}_{n}$. A lattice $L \subset \mathbb{Z}^{n}$ determines the group $\mathcal{G}=\mathbb{Z}^{n} / L$ and a distinguished set of $n$ generators $\mathbf{g}_{i}=\mathbf{e}_{i}+L$ for every $i=1, \ldots, n$, where $\mathbf{e}_{1}, \ldots, \mathbf{e}_{n}$ are the unit vectors of $\mathbb{Z}^{n}$.

Multigradings of polynomial rings have been extensively studied and systematically used over the last years, see [13] chapter 4, [14] chapter 8, [16] chapter 10. Several times one has to consider coarser gradings for an $S$ module than the finest one, see [5], 8], [12]. This procedure of passing from a finer to a coarser grading is called specialization or coarsening the grading, see [12]. This is the case studied in the present paper. We are interested in

1991 Mathematics Subject Classification. 14M25, 13F55.

Key words and phrases. Lattice ideals, multigradings, arithmetical rank, simplicial complex. 
the problem of computing the arithmetical rank of a toric or lattice ideal. The arithmetical rank, denoted by $\operatorname{ara}(J)$, of an ideal $J \subset K\left[x_{1}, \ldots, x_{n}\right]$ is the smallest integer $s$ for which there exist polynomials $F_{1}, \ldots, F_{s}$ in $J$ such that $\operatorname{rad}(J)=\operatorname{rad}\left(F_{1}, \ldots, F_{s}\right)$. The computation of the arithmetical rank of a lattice ideal $I_{L}$ is a difficult problem and remains open even in very simple cases like the ideal of the Macaulay curve in the three dimensional projective space, see [4] chapter 15. Every lattice ideal $I_{L}$ has a natural multigraded structure ([3, [16]), in fact it is $\mathcal{G}$-homogeneous for $\mathcal{G}=\mathbb{Z}^{n} / L$. The lattice ideal $\operatorname{rad}\left(I_{L}\right)$ can always be generated up to radical by $\mathcal{G}$-homogeneous polynomials, and sometimes this is possible with $\operatorname{ara}\left(I_{L}\right)$ such polynomials, as was shown in [6], [8]. But this is not the case in general. In an example of a lattice ideal studied in [9] the arithmetical rank is somewhere between 80 to 90 while the minimum number of $\mathcal{G}$-homogeneous polynomials needed to generate $\operatorname{rad}\left(I_{L}\right)$ up to radical is exactly 1740 . This means that $\mathcal{G}$-homogeneous polynomials are not always enough to minimally generate the radical of a lattice ideal up to radical. Therefore one has to better understand non $\mathcal{G}$-homogeneous set-theoretic intersections for lattice ideals. A first step in this direction is to consider coarser $\mathcal{F}$-gradings than the $\mathcal{G}$-grading and study the minimum number of $\mathcal{F}$-homogeneous polynomials needed to generate the radical of a lattice ideal up to radical. We will define a relation $\leq$ on the set of gradings by groups with $n$ generators. The grading defined by a group $\mathcal{F}=<\mathbf{f}_{1}, \ldots, \mathbf{f}_{n}>$ is called a specialization of $\mathcal{G}=<\mathbf{g}_{1}, \ldots, \mathbf{g}_{n}>$ if every $\mathcal{G}$-homogeneous ideal in $K\left[x_{1}, \ldots, x_{n}\right]$ is also $\mathcal{F}$-homogeneous and this will be denoted by $\mathcal{F} \leq \mathcal{G}$. Specializations of $\mathcal{G}$-gradings were used in 8 to compute concrete polynomial equations that set-theoretically define certain toric varieties. Section 2 of the paper is devoted to a more systematic study of specializations of $\mathcal{G}$-gradings.

Let $\mathcal{F}$ be a specialization of $\mathcal{G}$. Given a $\mathcal{G}$-homogeneous ideal $J \subset$ $K\left[x_{1}, \ldots, x_{n}\right]$, it is natural to define the $\mathcal{F}$-homogeneous arithmetical rank of $J$, denoted by $\operatorname{ara}_{\mathcal{F}}(J)$, as the smallest integer $s$ such that $\operatorname{rad}(J)=$ $\operatorname{rad}\left(F_{1}, \ldots, F_{s}\right)$ and all the polynomials $F_{1}, \ldots, F_{s}$ are $\mathcal{F}$-homogeneous. This notion is important for two reasons:

(1) It is an upper bound for ara $(J)$. More precisely for a $\mathcal{G}$-homogeneous ideal $J$ and a specialization $\mathcal{F}$ of $\mathcal{G}$ it holds:

$$
\operatorname{ht}(J) \leq \operatorname{ara}(J) \leq \operatorname{ara}_{\mathcal{F}}(J) \leq \operatorname{ara}_{\mathcal{G}}(J),
$$

where $\operatorname{ht}(J)$ is the height of $J$. When $\operatorname{ht}(J)=\operatorname{ara}(J)$ the ideal $J$ is called set-theoretic complete intersection and when $h t(J)=\operatorname{ara}_{\mathcal{F}}(J)$ it is called $\mathcal{F}$-homogeneous set-theoretic complete intersection.

(2) For every $\mathcal{G}$-homogeneous ideal $J$ there is an $\mathcal{F}$-grading such that $\operatorname{ara}(J)=\operatorname{ara}_{\mathcal{F}}(J)$ and $\mathcal{F} \leq \mathcal{G}$, see Proposition 3.3.

The most difficult part in computing the arithmetical rank or $\mathcal{F}$-homogeneous arithmetical rank of a lattice ideal $I_{L_{\mathcal{G}}}$ is to find sharp lower bounds. Lower bounds of the arithmetical rank of $I_{L_{\mathcal{G}}}$ can be provided sometimes by local or etalé cohomology, see [1, 2]. The main result of this article, Theorem 3.10 , generalizes the results of [9, [10] and provides lower bounds for the $\mathcal{F}$-homogeneous arithmetical rank of the lattice ideal $I_{L_{\mathcal{G}}}$, where $\mathcal{F}$ is a specialization of $\mathcal{G}$, using combinatorial invariants of a simplicial complex 
associated to the ideal $I_{L_{\mathcal{G}}}$ and the specialization $\mathcal{F}$ of $\mathcal{G}$. As an application in Section 4 we study an example of a lattice ideal $I_{L_{\mathcal{G}}}$. We compute the bounds given in Theorem 3.10 and prove that they are sharp. Finally we show that the lattice ideal $I_{L_{\mathcal{G}}}$ is not a $\mathcal{F}$-homogeneous set-theoretic complete intersection for infinitely many specializations $\mathcal{F}$ of $\mathcal{G}$.

\section{BASIC THEORY OF SPECIALIZATIONS OF $\mathcal{G}$-GRADINGS}

2.1. Preliminaries. Given a lattice $L \subset \mathbb{Z}^{n}$, the ideal

$$
I_{L}:=\left(\left\{\mathbf{x}^{\alpha_{+}}-\mathbf{x}^{\alpha_{-}} \mid \alpha=\alpha_{+}-\alpha_{-} \in L\right\}\right) \subset K\left[x_{1}, \ldots, x_{n}\right]
$$

is called lattice ideal. Here $\alpha_{+} \in \mathbb{N}^{n}$ and $\alpha_{-} \in \mathbb{N}^{n}$ denote the positive and negative part of $\alpha$, respectively, and $\mathbf{x}^{\beta}=x_{1}^{b_{1}} \cdots x_{n}^{b_{n}}$ for $\beta=\left(b_{1}, \ldots, b_{n}\right) \in$ $\mathbb{N}^{n}$. The saturation of a sublattice $L$ of $\mathbb{Z}^{n}$ is the lattice

$$
\operatorname{Sat}(L):=\left\{\alpha \in \mathbb{Z}^{n} \mid d \alpha \in L \text { for some } d \in \mathbb{Z}^{*}\right\} .
$$

We say that the lattice $L$ is saturated if $L=\operatorname{Sat}(L)$. This is equivalent to saying that the group $\mathbb{Z}^{n} / L$ is torsion free. The lattice ideal $I_{L}$ is prime if and only if $L$ is saturated. A prime lattice ideal is called a toric ideal, while the set of zeroes in $K^{n}$ is an affine toric variety in the sense of [16].

If $L=<\mathbf{l}_{1}, \ldots, \mathbf{l}_{k}>$ is a sublattice of $\mathbb{Z}^{n}$ of rank $k<n$, then there exists a set of vectors $A=\left\{\mathbf{a}_{1}, \ldots, \mathbf{a}_{n}\right\} \subset \mathbb{Z}^{m}$ such that $\operatorname{Sat}(L)=L_{\mathbb{Z} A}$, where $m=n-k$ and $\mathbb{Z} A=\left\{q_{1} \mathbf{a}_{1}+\cdots+q_{n} \mathbf{a}_{n}: q_{1}, \ldots, q_{n} \in \mathbb{Z}\right\}$ is the lattice spanned by $A$. Remark that $L_{\mathbb{Z} A}$ is saturated. In order to determine $A$ we work as follows. Set $\mathbf{L}=\left(\mathbf{l}_{1}, \ldots, \mathbf{l}_{k}\right)$ the matrix with columns $\mathbf{l}_{1}, \ldots, \mathbf{l}_{k}$, then there are unimodular integral matrices $\mathbf{U}$ and $\mathbf{Q}$ of orders $n$ and $k$, respectively, such that $\mathbf{U L Q}=\operatorname{diag}\left(\lambda_{1}, \ldots, \lambda_{k}, 0, \ldots, 0\right)$ is in Smith normal form. Here $\lambda_{1}, \ldots, \lambda_{k}$ are natural numbers and $\lambda_{i}$ divides $\lambda_{i+1}$. The set $A$ can be chosen as the one consisting of the columns of the matrix formed by the last $n-k$ rows of $\mathbf{U}$. Moreover the group $\mathbb{Z}^{n} / L$ is isomorphic to $\mathbb{Z}^{m} \oplus \mathbb{Z}_{\lambda_{1}} \oplus \cdots \oplus \mathbb{Z}_{\lambda_{k}}$, [14. We can associate with the lattice ideal $I_{L}$ the rational polyhedral cone

$$
\sigma_{A}:=\operatorname{pos}_{\mathbb{Q}}(A)=\left\{\sum_{i=1}^{n} d_{i} \mathbf{a}_{i} \mid d_{i} \in \mathbb{Q}_{\geq 0}\right\} .
$$

A face of $\sigma_{A}$ is any set of the form

$$
\mathcal{T}=\sigma_{A} \cap\left\{\mathbf{x} \in \mathbb{Q}^{m}: \mathbf{c x}=0\right\}
$$

where $\mathbf{c} \in \mathbb{Q}^{m}$ and $\mathbf{c x} \geq 0$ for all $\mathbf{x} \in \sigma_{A}$. Faces of dimension one are called extreme rays. A cone $\sigma_{A}$ is strongly convex if $\{\mathbf{0}\}$ is a face of $\sigma_{A}$, where $\mathbf{0}=(0, \ldots, 0)$.

2.2. Specializations of $\mathcal{G}$-gradings. The next theorem indicates that the specialization property reflects on the lattice of relations of the generators of $\mathcal{G}$ and correspondingly in the lattice ideal $I_{L_{\mathcal{G}}}$.

Theorem 2.1. Let $\mathcal{F}=<\mathbf{f}_{1}, \ldots, \mathbf{f}_{n}>$ and $\mathcal{G}=<\mathbf{g}_{1}, \ldots, \mathbf{g}_{n}>$ be finitely generated abelian groups. The following are equivalent:

(a) $\mathcal{F} \leq \mathcal{G}$, i.e. $\mathcal{F}$ is a specialization of $\mathcal{G}$. 
(b) $L_{\mathcal{G}} \subset L_{\mathcal{F}}$.

(c) $I_{L_{\mathcal{G}}} \subset I_{L_{\mathcal{F}}}$.

(d) There is a group epimorphism from $\mathcal{G}$ to $\mathcal{F}$, sending $\mathbf{g}_{i}$ to $\mathbf{f}_{i}$.

Proof. The equivalence $(\mathrm{b}) \Leftrightarrow(\mathrm{c})$ is easily derived from the fact that a binomial $\mathbf{x}^{\mathbf{u}}-\mathbf{x}^{\mathbf{v}}$ belongs to a lattice ideal $I_{L_{\mathcal{G}}}$ if and only if the vector $\mathbf{u}-\mathbf{v}$ belongs to $L_{\mathcal{G}}$. We will prove that (a) $\Leftrightarrow$ (b).

(a) $\Rightarrow$ (b) Let $\mathbf{u}=\mathbf{u}_{+}-\mathbf{u}_{-} \in L_{\mathcal{G}}$, where $\mathbf{u}_{+}=\left(u_{+, 1}, \ldots, u_{+, n}\right)$ and $\mathbf{u}_{-}=$ $\left(u_{-, 1}, \ldots, u_{-, n}\right)$. Then

$$
u_{+, 1} \mathbf{g}_{1}+\cdots+u_{+, n} \mathbf{g}_{n}=u_{-, 1} \mathbf{g}_{1}+\cdots+u_{-, n} \mathbf{g}_{n} .
$$

The ideal $J=\left(\mathbf{x}^{\mathbf{u}_{+}}-\mathbf{x}^{\mathbf{u}_{-}}\right)$is $\mathcal{G}$-homogeneous, so it is also $\mathcal{F}$-homogeneous. Thus

$$
u_{+, 1} \mathbf{f}_{1}+\cdots+u_{+, n} \mathbf{f}_{n}=u_{-, 1} \mathbf{f}_{1}+\cdots+u_{-, n} \mathbf{f}_{n}
$$

and therefore $\mathbf{u} \in L_{\mathcal{F}}$.

(b) $\Rightarrow$ (a) Let $J \subset K\left[x_{1}, \ldots, x_{n}\right]$ be a $\mathcal{G}$-homogeneous ideal and $\mathbf{x}^{\mathbf{u}}, \mathbf{x}^{\mathbf{v}}$ two monomials of a $\mathcal{G}$-homogeneous generator $F$ of $J$, where $\mathbf{u}=\left(u_{1}, \ldots, u_{n}\right)$ and $\mathbf{v}=\left(v_{1}, \ldots, v_{n}\right)$. We have

$$
u_{1} \mathbf{g}_{1}+\cdots+u_{n} \mathbf{g}_{n}=v_{1} \mathbf{g}_{1}+\cdots+v_{n} \mathbf{g}_{n},
$$

which implies that the vector $\mathbf{w}=\left(u_{1}-v_{1}, \ldots, u_{n}-v_{n}\right)$ belongs to $L_{\mathcal{G}}$. But $L_{\mathcal{G}} \subset L_{\mathcal{F}}$, so w belongs to $L_{\mathcal{F}}$ and therefore

$$
u_{1} \mathbf{f}_{1}+\cdots+u_{n} \mathbf{f}_{n}=v_{1} \mathbf{f}_{1}+\cdots+v_{n} \mathbf{f}_{n} .
$$

Thus $J$ is $\mathcal{F}$-homogeneous.

Finally we will prove that (b) $\Leftrightarrow(\mathrm{d})$. Assume first that $L_{\mathcal{G}} \subset L_{\mathcal{F}}$. We define $\phi: \mathcal{G} \rightarrow \mathcal{F}$ by setting

$$
\phi\left(\alpha_{1} \mathbf{g}_{1}+\cdots+\alpha_{n} \mathbf{g}_{n}\right)=\alpha_{1} \mathbf{f}_{1}+\cdots+\alpha_{n} \mathbf{f}_{n} .
$$

The map $\phi$ is well defined. Let $\mathbf{u} \in \mathcal{G}$ be such that $\mathbf{u}=\alpha_{1} \mathbf{g}_{1}+\cdots+\alpha_{n} \mathbf{g}_{n}$ and $\mathbf{u}=\beta_{1} \mathbf{g}_{1}+\cdots+\beta_{n} \mathbf{g}_{n}$. Then the vector $\left(\alpha_{1}-\beta_{1}, \ldots, \alpha_{n}-\beta_{n}\right)$ belongs to $L_{\mathcal{G}}$, which is a subset of $L_{\mathcal{F}}$ and therefore $\alpha_{1} \mathbf{f}_{1}+\cdots+\alpha_{n} \mathbf{f}_{n}=\beta_{1} \mathbf{f}_{1}+\cdots+\beta_{n} \mathbf{f}_{n}$. Obviously $\phi$ is a homomorphism mapping $\mathcal{G}$ onto $\mathcal{F}$.

Conversely assume that there is a group epimorphism $\phi: \mathcal{G} \rightarrow \mathcal{F}$, sending $\mathbf{g}_{i}$ to $\mathbf{f}_{i}$. Let $\mathbf{u}=\left(u_{1}, \ldots, u_{n}\right) \in L_{\mathcal{G}}$, then $u_{1} \mathbf{g}_{1}+\cdots+u_{n} \mathbf{g}_{n}=\mathbf{0}_{\mathcal{G}}$ and therefore $\phi\left(u_{1} \mathbf{g}_{1}+\cdots+u_{n} \mathbf{g}_{n}\right)=\mathbf{0}_{\mathcal{F}}$. Thus $u_{1} \mathbf{f}_{1}+\cdots+u_{n} \mathbf{f}_{n}=\mathbf{0}_{\mathcal{F}}$, which implies that u belongs to $L_{\mathcal{F}}$.

Remark 2.2. Let $\phi$ be the epimorphism defined in the proof of Theorem 2.1. Any $\mathcal{G}$-graded $S$-module $M$ can be regarded as an $\mathcal{F}$-graded module by setting $M_{\mathbf{u}}=\bigoplus_{\mathbf{v} \in \phi^{-1}(\mathbf{u})} M_{\mathbf{v}}$.

Corollary 2.3. Let $\mathcal{F}, \mathcal{G}$ be finitely generated abelian groups with n generators and $A, B$ sets of vectors such that $\operatorname{Sat}\left(L_{\mathcal{F}}\right)=L_{\mathbb{Z} B}$ and $\operatorname{Sat}\left(L_{\mathcal{G}}\right)=L_{\mathbb{Z} A}$. If $\mathcal{F}$ is a specialization of $\mathcal{G}$, then $\mathbb{Z} B$ is a specialization of $\mathbb{Z} A$.

Proof. From Theorem 2.1 we have that $L_{\mathcal{G}} \subset L_{\mathcal{F}}$ and therefore $\operatorname{Sat}\left(L_{\mathcal{G}}\right) \subset$ $\operatorname{Sat}\left(L_{\mathcal{F}}\right)$. Thus $L_{\mathbb{Z} A} \subset L_{\mathbb{Z} B}$, so, again from Theorem 2.1, the group $\mathbb{Z} B$ is a specialization of $\mathbb{Z} A$. 
Remark 2.4. The group $\mathbb{Z} B$ is a specialization of $\mathcal{F}$, since $L_{\mathcal{F}} \subset \operatorname{Sat}\left(L_{\mathcal{F}}\right)$, and similarly $\mathbb{Z} A$ is a specialization of $\mathcal{G}$.

Let $\widehat{\pi}: \mathbb{Q}^{m} \rightarrow \mathbb{Q}^{r}$ be a rational affine map with $\widehat{\pi}\left(\sigma_{A}\right)=\sigma_{B}$. The restriction

$$
\pi:=\left.\widehat{\pi}\right|_{\sigma_{A}}: \sigma_{A} \rightarrow \sigma_{B}
$$

is called projection of cones.

Proposition 2.5. If $\mathbb{Z} B$ is a specialization of $\mathbb{Z} A$, for $A=\left\{\mathbf{a}_{1}, \ldots, \mathbf{a}_{n}\right\}$ and $B=\left\{\mathbf{b}_{1}, \ldots, \mathbf{b}_{n}\right\}$, then there is a projection of cones $\pi: \sigma_{A} \rightarrow \sigma_{B}$ given by $\pi\left(\mathbf{a}_{i}\right)=\mathbf{b}_{i}$ for all $i=1, \ldots, n$.

Proof. From Theorem 2.1 we have that $L_{\mathbb{Z} A} \subset L_{\mathbb{Z} B}$, since $\mathbb{Z} B$ is a specialization of $\mathbb{Z} A$, so, from Theorem 2.2 in [8], there is a projection of cones $\pi: \sigma_{A} \rightarrow \sigma_{B}$ given by $\pi\left(\mathbf{a}_{i}\right)=\mathbf{b}_{i}$ for all $i=1, \ldots, n$.

We say that $\mathcal{F}$ is equivalent to $\mathcal{G}$, denoted by $\mathcal{F} \sim \mathcal{G}$, if every $\mathcal{F}$-homogeneous ideal is also $\mathcal{G}$-homogeneous and conversely.

Corollary 2.6. Let $\mathcal{F}=<\mathbf{f}_{1}, \ldots, \mathbf{f}_{n}>$ and $\mathcal{G}=<\mathbf{g}_{1}, \ldots, \mathbf{g}_{n}>$ be finitely generated abelian groups. The following are equivalent:

(a) $\mathcal{F} \sim \mathcal{G}$.

(b) $I_{L_{\mathcal{F}}}=I_{L_{\mathcal{G}}}$.

(c) $L_{\mathcal{F}}=L_{\mathcal{G}}$.

(d) $\mathcal{F}, \mathcal{G}$ are isomorphic groups and the isomorphism sends $\mathbf{g}_{i}$ to $\mathbf{f}_{i}$.

Although equivalent gradings defined by different groups provide exactly the same grading in the polynomial ring, it is interesting to study them for other reasons, including the fact that they give different toric sets, see [11, which has applications to Algebraic Statistics, see [7].

From now on $\widetilde{\mathcal{G}}$ will denote the equivalence class of the group $\mathcal{G}$. By writing $\widetilde{\mathcal{F}} \leq \widetilde{\mathcal{G}}$ we mean that for every pair of representatives $\mathcal{F}$ and $\mathcal{G}$ of $\widetilde{\mathcal{F}}$ and $\widetilde{\mathcal{G}}$, respectively, it holds $\mathcal{F} \leq \mathcal{G}$. From Theorem 2.1 it is easily derived that if $\mathcal{F} \leq \mathcal{G}$ and $\mathcal{G} \leq \mathcal{H}$, then $\mathcal{F} \leq \mathcal{H}$. So $\leq$ is a partial order on the set of equivalence classes of gradings of groups with $n$ generators with respect to relation $\sim$. Let $\mathcal{F}$ and $\mathcal{G}$ be groups generated by $n$ elements. We define the join of $\widetilde{\mathcal{F}}$ and $\widetilde{\mathcal{G}}$, denoted by $\widetilde{\mathcal{F} \vee \mathcal{G}}$, to be the equivalence class of the group $\mathbb{Z}^{n} /\left(L_{\mathcal{F}} \cap L_{\mathcal{G}}\right)$. The meet of $\widetilde{\mathcal{F}}$ and $\widetilde{\mathcal{G}}$, denoted by $\widetilde{\mathcal{F} \wedge \mathcal{G}}$, is defined as the equivalence class of the group $\mathbb{Z}^{n} /\left(L_{\mathcal{F}}+L_{\mathcal{G}}\right)$. We have that $\mathcal{F} \wedge \mathcal{G} \leq \mathcal{F}$ and $\mathcal{F} \wedge \mathcal{G} \leq \mathcal{G}$, since $L_{\mathcal{F}}+L_{\mathcal{G}}$ contains both $L_{\mathcal{F}}, L_{\mathcal{G}}$. Moreover if $\mathcal{H} \leq \mathcal{F}$ and $\mathcal{H} \leq \mathcal{G}$, then $\mathcal{H} \leq \mathcal{F} \wedge \mathcal{G}$ since $L_{\mathcal{F}}+L_{\mathcal{G}}$ is the smallest sublattice of $\mathbb{Z}^{n}$ containing $L_{\mathcal{F}}$ and $L_{\mathcal{G}}$. The finest grading is given by the abelian group $\mathbb{Z}^{n}$ with generators the vectors $\mathbf{e}_{i}=(0, \ldots, 0,1,0, \ldots, 0)$, where the 1 is in the $i$ th position. Note that every finitely generated abelian group $\mathcal{G}$ is a specialization of $\mathbb{Z}^{n}$, since $L_{\mathbb{Z}^{n}}=<\mathbf{0}>$. The only $\mathbb{Z}^{n}$-homogeneous ideals in $K\left[x_{1}, \ldots, x_{n}\right]$ are the monomial ideals, while the coarsest grading is given by the zero group $\mathcal{O}$ generated by the set of $n$ zero vectors $\mathbf{o}_{i}=\mathbf{0}$. Note that $\mathcal{O}$ is a specialization of every abelian group $\mathcal{G}$ with $n$ generators and $I_{L_{\mathcal{O}}}=<x_{1}-1, \ldots, x_{n}-1>$. Every ideal in $K\left[x_{1}, \ldots, x_{n}\right]$ is $\mathcal{O}$-homogeneous. So actually $\mathcal{O} \leq \mathcal{G} \leq \mathbb{Z}^{n}$. 
We say that a $\mathcal{G}$-grading is positive if $L_{\mathcal{G}} \cap \mathbb{N}^{n}=\{\mathbf{0}\}$. This is equivalent to saying that the rational polyhedral cone $\sigma_{A}$ is strongly convex. Specializations can be used to give an equivalent characterization of the positivity condition. For more equivalent conditions, see [14, Chapter 8.

Theorem 2.7. Let $\mathcal{G}=<\mathbf{g}_{1}, \ldots, \mathbf{g}_{n}>$ be a finitely generated abelian group. The $\mathcal{G}$-grading is positive if and only if there exists a set $M=\left\{m_{1}, \ldots, m_{n}\right\}$ of positive integers such that $\mathbb{Z} M$ is a specialization of $\mathcal{G}$.

Proof. Suppose first that there is a set $M=\left\{m_{1}, \ldots, m_{n}\right\}$ with $\mathbb{Z} M \leq \mathcal{G}$ and that the $\mathcal{G}$-grading is not positive. Then there is a relation

$$
\lambda_{1} \mathbf{g}_{1}+\cdots+\lambda_{n} \mathbf{g}_{n}=\mathbf{0}_{\mathcal{G}},
$$

where every $\lambda_{i} \in \mathbb{Z}$ is non negative and there is at least one $\lambda_{j}$ different from zero. Let $\phi: \mathcal{G} \rightarrow \mathbb{Z} M$ be the group epimorphism, sending $\mathbf{g}_{i}$ to $m_{i}$. We have that $\phi\left(\lambda_{1} \mathbf{g}_{1}+\cdots+\lambda_{n} \mathbf{g}_{n}\right)=0$, so $\lambda_{1} \phi\left(\mathbf{g}_{1}\right)+\cdots+\lambda_{n} \phi\left(\mathbf{g}_{n}\right)=0$ and therefore $\lambda_{1} m_{1}+\cdots+\lambda_{n} m_{n}=0$. But $\lambda_{1} m_{1}+\cdots+\lambda_{n} m_{n}>0$, since $m_{1}, \ldots, m_{n}$ are positive integers and $\lambda_{i}$ are non negative with at least one of them different from zero, a contradiction. Suppose now that the $\mathcal{G}$-grading is positive, this means that $\mathbf{0}$ is a face of the corresponding rational polyhedral cone $\sigma_{A}$. Thus there is a defining vector $\mathbf{c}_{\boldsymbol{0}}$ of the above face such that $\mathbf{c}_{0} \mathbf{a}_{i}>0$, for every $i=1, \ldots, n$. Set $m_{i}=\mathbf{c}_{0} \mathbf{a}_{i}$, for $i=1, \ldots, n$, then $M=\mathbb{Z}\left\{m_{1}, \ldots, m_{n}\right\}$ is specialization of $\mathbb{Z} A$. Let $\mathbf{u}=\left(u_{1}, \ldots, u_{n}\right) \in L_{\mathbb{Z} A}$, then $u_{1} \mathbf{a}_{1}+\cdots+u_{n} \mathbf{a}_{n}=\mathbf{0}$. So $\mathbf{c}_{\mathbf{0}}\left(u_{1} \mathbf{a}_{1}+\cdots+u_{n} \mathbf{a}_{n}\right)=0$ and therefore $u_{1}\left(\mathbf{c}_{0} \mathbf{a}_{1}\right)+\cdots+u_{n}\left(\mathbf{c}_{0} \mathbf{a}_{n}\right)=0$. Thus $L_{\mathbb{Z} A} \subset L_{\mathbb{Z} M}$. From Remark 2.4 we deduce that $\mathbb{Z} M$ is a specialization of $\mathcal{G}$.

Note that if $\mathcal{F}$ is a specialization of $\mathcal{G}$ and the $\mathcal{F}$-grading is positive, then the $\mathcal{G}$-grading is positive.

\section{Arithmetical RANK of Lattice ideals}

In this section the first goal is to prove the existence of an $\mathcal{H}$-grading such that $\operatorname{ara}\left(I_{L_{\mathcal{G}}}\right)=\operatorname{ara\mathcal {H}}\left(I_{L_{\mathcal{G}}}\right)$. After that we will assign to every pair $(\mathcal{F}, \mathcal{G})$ a simplicial complex $\mathcal{D}_{\mathcal{F}}^{\mathcal{G}}$ and to every polynomial $F \in K\left[x_{1}, \ldots, x_{n}\right]$ a subcomplex of $\mathcal{D}_{\mathcal{F}}^{\mathcal{G}}$, where $\mathcal{F}$ is a specialization of $\mathcal{G}$. The second goal is to prove that if $F_{1}, \ldots, F_{s}$ are $\mathcal{F}$-homogeneous polynomials and generate $\operatorname{rad}\left(I_{L_{\mathcal{G}}}\right)$ up to radical, then each of the subcomplexes corresponding to the polynomials $F_{i}$ is a simplex and their union is a spanning subcomplex of $\mathcal{D}_{\mathcal{F}}$. This will enable us to provide lower bounds for the $\mathcal{F}$-homogeneous arithmetical rank based on combinatorial invariants of the simplicial complex $\mathcal{D}_{\mathcal{F}}^{\mathcal{G}}$.

Theorem 3.1. Let $\left\{F_{1}, \ldots, F_{s}\right\}$ be a set of polynomials in $K\left[x_{1}, \ldots, x_{n}\right]$. There exists a finest $\mathcal{F}$-grading such that all $F_{1}, \ldots, F_{s}$ are $\mathcal{F}$-homogeneous. This grading is unique up to equivalence.

Proof. Every polynomial $F_{i} \neq 0$ can be written as a finite sum of terms, i.e. $F_{i}=\sum_{j} c_{i j} \mathbf{x}^{\mathbf{u}_{j}^{i}}$ where $K \ni c_{i j} \neq 0$. Let $L$ be the lattice generated by all the vectors $\mathbf{u}_{j}^{i}-\mathbf{u}_{1}^{i}$, for every $i=1, \ldots, s$. The polynomials $F_{1}, \ldots, F_{s}$ 
are $\mathcal{F}$-homogeneous for $\mathcal{F}=\mathbb{Z}^{n} / L$. It remains to prove that $\mathcal{F}$ is the finest. Suppose that $F_{1}, \ldots, F_{s}$ are also $\mathcal{G}$-homogeneous, then, for every $i=1, \ldots, s$, we have that $\operatorname{deg}_{\mathcal{G}}\left(\mathbf{u}_{j}^{i}\right)=\operatorname{deg}_{\mathcal{G}}\left(\mathbf{u}_{1}^{i}\right)$. So the vectors $\mathbf{u}_{j}^{i}-\mathbf{u}_{1}^{i}$ belong to $L_{\mathcal{G}}$ and therefore $L_{\mathcal{F}} \subset L_{\mathcal{G}}$. Thus $\mathcal{G} \leq \mathcal{F}$. This fact also implies that $\mathcal{F}$ is unique up to equivalence.

Corollary 3.2. Let $\left\{F_{1}, \ldots, F_{s}\right\}$ be a set of polynomials in $K\left[x_{1}, \ldots, x_{n}\right]$ and let $\mathcal{G}=<\mathbf{g}_{1}, \ldots, \mathbf{g}_{n}>$ be a finitely generated abelian group. There exists a finest $\mathcal{H}$-grading such that all $F_{1}, \ldots, F_{s}$ are $\mathcal{H}$-homogeneous and also $\mathcal{H} \leq \mathcal{G}$. This grading is unique up to equivalence.

Proof. From Theorem 3.1 there exists a finest $\mathcal{F}$-grading such that the polynomials $F_{1}, \ldots, F_{s}$ are $\mathcal{F}$-homogeneous. This grading is unique up to equivalence. Let $\mathcal{H}=\mathcal{F} \wedge \mathcal{G}$ be any representative of the class $\widetilde{\mathcal{F} \wedge \mathcal{G}}$, then $\mathcal{H} \leq \mathcal{G}$. Moreover $F_{1}, \ldots, F_{s}$ are $\mathcal{H}$-homogeneous, since the ideal generated by $F_{1}, \ldots, F_{s}$ is $\mathcal{F}$-homogeneous and $\mathcal{H} \leq \mathcal{F}$. To prove that $\mathcal{H}$ is the finest, assume that the $F_{1}, \ldots, F_{s}$ are $\mathcal{M}$-homogeneous and also $\mathcal{M} \leq \mathcal{G}$. Then $\mathcal{M} \leq \mathcal{F}$, so $\mathcal{M} \leq \mathcal{F} \wedge \mathcal{G}=\mathcal{H}$.

Proposition 3.3. For any $\mathcal{G}$-homogeneous ideal $J \subset K\left[x_{1}, \ldots, x_{n}\right]$ there is a finest $\mathcal{H}$-grading such that

(1) $J$ is $\mathcal{H}$-homogeneous

(2) $\mathcal{H} \leq \mathcal{G}$ and

(3) $\operatorname{ara}(J)=\operatorname{ara}_{\mathcal{H}}(J)$.

This grading is unique up to equivalence.

Proof. Let $\operatorname{ara}(J)=s$, which implies that $\operatorname{rad}(J)=\operatorname{rad}\left(F_{1}, \ldots, F_{s}\right)$ for some polynomials $F_{1}, \ldots, F_{s}$ in $K\left[x_{1}, \ldots, x_{n}\right]$. From Corollary 3.2 there exists a finest $\mathcal{H}$-grading such that $F_{1}, \ldots, F_{s}$ are $\mathcal{H}$-homogeneous and $\mathcal{H} \leq$ $\mathcal{G}$. This grading is unique up to equivalence. It follows that $\operatorname{ara}(J)=$ $\operatorname{ara}_{\mathcal{H}}(J)$.

The next theorem is an easy consequence of Proposition 3.3, since every lattice ideal $I_{L_{\mathcal{G}}}$ is $\mathcal{G}$-homogeneous.

Theorem 3.4. For any lattice ideal $I_{L_{\mathcal{G}}} \subset K\left[x_{1}, \ldots, x_{n}\right]$ there is a unique up to equivalence finest $\mathcal{H}$-grading such that

(1) $I_{L_{\mathcal{G}}}$ is $\mathcal{H}$-homogeneous

(2) $\mathcal{H} \leq \mathcal{G}$ and

(3) $\operatorname{ara}\left(I_{L_{\mathcal{G}}}\right)=\operatorname{ara} \mathcal{H}\left(I_{L_{\mathcal{G}}}\right)$.

Generally it is difficult to compute a priori the grading $\mathcal{H}$ of Theorem 3.4. But using the theory of simplicial complexes we can find bounds for the $\mathcal{F}$-homogeneous arithmetical rank of a lattice ideal $I_{L}$, in the case where the grading induced by the lattice $L$ is positive. Also note that in several cases one expects that the group $\mathcal{H}$ of Theorem 3.4 coincides with $\mathcal{O}$. But even in this case one gets interesting results from the simplicial complex $\mathcal{D}_{\mathcal{G}}^{\mathcal{G}}$, see Definition 3.5, such as a lower bound on the number of monomials in the support of the polynomials that define the radical, but also to the number of $\mathcal{F}$-homogeneous components, for various $\mathcal{F}$ 's.

Let $\mathcal{G}$ be a finitely generated abelian group with $n$ generators and $\sigma_{A}$ the rational polyhedral cone associated with the lattice ideal $I_{L_{\mathcal{G}}} \subset K\left[x_{1}, \ldots, x_{n}\right]$, 
for an appropriate set of vectors $A=\left\{\mathbf{a}_{1}, \ldots, \mathbf{a}_{n}\right\}$. From now on we shall write $\sigma_{\mathcal{G}}$ instead of $\sigma_{A}$. The relative interior of $\sigma_{\mathcal{G}}$, denoted by relint $t_{\mathbb{Q}}\left(\sigma_{\mathcal{G}}\right)$, is the set of all positive rational linear combinations of $\mathbf{a}_{1}, \ldots, \mathbf{a}_{n}$. When $\sigma_{\mathcal{G}}$ is strongly convex we have that $\sigma_{\mathcal{G}}=\operatorname{pos}_{\mathbb{Q}}\left(\mathbf{r}_{1}, \ldots, \mathbf{r}_{t}\right)$, where $\left\{\mathbf{r}_{1}, \ldots, \mathbf{r}_{t}\right\}$ is a set of integer vectors, one for each extreme ray of $\sigma_{\mathcal{G}}$. The vectors $\mathbf{r}_{i}$ are called extreme vectors of $\sigma_{\mathcal{G}}$. Given a subset $E$ of $\{1, \ldots, t\}$ we denote by $\sigma_{\mathcal{G}}(E)$ the subcone $\operatorname{pos}_{\mathbb{Q}}\left(\mathbf{r}_{i} \mid i \in E\right)$ of $\sigma_{\mathcal{G}}$. We are going to deal only with subcones $\sigma_{\mathcal{G}}(E)$, which are not faces of the cone $\sigma_{\mathcal{G}}$. They form a poset ordered by inclusion. Let $\left\{\sigma_{\mathcal{G}}\left(\mathbb{E}_{1}\right), \ldots, \sigma_{\mathcal{G}}\left(\mathbb{E}_{f}\right)\right\}$ be the minimal elements of this poset, which are called the minimal non faces of $\sigma_{\mathcal{G}}$. To every specialization we assign a simplicial complex $\mathcal{D}_{\mathcal{F}}^{\mathcal{G}}$ that generalizes the complex $\Delta_{\sigma}=\mathcal{D}_{\mathcal{G}}^{\mathcal{G}}$ defined in [9] and [10].

Definition 3.5. Let $\mathcal{F}$ be a specialization of $\mathcal{G}$ and $\pi: \sigma_{\mathcal{G}} \rightarrow \sigma_{\mathcal{F}}$ the corresponding projection of cones. We define $\mathcal{D}_{\mathcal{F}}^{\mathcal{G}}$ to be the simplicial complex with vertices $\left\{\mathbb{E}_{1}, \ldots, \mathbb{E}_{f}\right\}$ such that $T \subset\left\{\mathbb{E}_{1}, \ldots, \mathbb{E}_{f}\right\}$ belongs to $\mathcal{D}_{\mathcal{F}}^{\mathcal{G}}$ if and only if

$$
\bigcap_{\mathbb{E}_{i} \in T} \operatorname{relint}_{\mathbb{Q}}\left(\pi\left(\sigma_{\mathcal{G}}\left(\mathbb{E}_{i}\right)\right)\right) \neq \emptyset .
$$

A subcomplex $H$ of a simplicial complex $\mathcal{D}$ is called a spanning subcomplex if both have exactly the same set of vertices. The following proposition shows that the simplicial complex $\mathcal{D}_{\mathcal{G}}^{\mathcal{G}}$ is a spanning subcomplex of $\mathcal{D}_{\mathcal{F}}^{\mathcal{G}}$.

Proposition 3.6. Let $\mathcal{F} \leq \mathcal{G}$ be finitely generated abelian groups with $n$ generators. Then

$$
\mathcal{D}_{\mathcal{G}}^{\mathcal{G}} \subset \mathcal{D}_{\mathcal{F}}^{\mathcal{G}} \subset \mathcal{D}_{\mathcal{O}}^{\mathcal{G}}
$$

where $\mathcal{O}$ is the group generated by the set of $n$ zero vectors $\mathbf{o}_{i}=\mathbf{0}$. In fact $\mathcal{D}_{\mathcal{G}}^{\mathcal{G}}$ is a spanning subcomplex of $\mathcal{D}_{\mathcal{F}}^{\mathcal{G}}$, the simplicial complex $\mathcal{D}_{\mathcal{F}}^{\mathcal{G}}$ is a spanning subcomplex of $\mathcal{D}_{\mathcal{O}}^{\mathcal{G}}$ and $\mathcal{D}_{\mathcal{O}}^{\mathcal{G}}$ is a simplex.

Proof. From the definitions of the three simplicial complexes, all of them have the same set of vertices. Let $T \in \mathcal{D}_{\mathcal{G}}^{\mathcal{G}}$, then

$$
\bigcap_{\mathbb{E}_{i} \in T} \operatorname{relint}_{\mathbb{Q}}\left(\sigma_{\mathcal{G}}\left(\mathbb{E}_{i}\right)\right) \neq \emptyset
$$

Hence there exists a $\mathbf{x} \in \bigcap_{\mathbb{E}_{i} \in T}$ relint $_{\mathbb{Q}}\left(\sigma_{\mathcal{G}}\left(\mathbb{E}_{i}\right)\right)$, which implies that $\pi(\mathbf{x})$ belongs to $\bigcap_{\mathbb{E}_{i} \in T}$ relint $_{\mathbb{Q}}\left(\pi\left(\sigma_{\mathcal{G}}\left(\mathbb{E}_{i}\right)\right)\right)$. Consequently

$$
\bigcap_{\mathbb{E}_{i} \in T} \operatorname{relint}_{\mathbb{Q}}\left(\pi\left(\sigma_{\mathcal{G}}\left(\mathbb{E}_{i}\right)\right)\right) \neq \emptyset \text {. }
$$

Thus $T \in \mathcal{D}_{\mathcal{F}}^{\mathcal{G}}$ and therefore $\mathcal{D}_{\mathcal{G}}^{\mathcal{G}} \subset \mathcal{D}_{\mathcal{F}}^{\mathcal{G}}$.

Let $A=\left\{\mathbf{a}_{1}, \ldots, \mathbf{a}_{n}\right\}$ be a set of vectors such that $\operatorname{Sat}\left(L_{\mathcal{G}}\right)=L_{\mathbb{Z} A}$ and let $\pi_{0}$ be the projection of cones sending $\mathbf{a}_{i}$ to $\mathbf{o}_{i}$. Then, for every $j=1, \ldots, f$, we have that $\pi_{0}\left(\sigma_{\mathcal{G}}\left(\mathbb{E}_{j}\right)\right)$ equals $\{\boldsymbol{0}\}$. So

$$
\bigcap_{\mathbb{E}_{i} \in T} \text { relint }_{\mathbb{Q}}(\{\mathbf{0}\}) \neq \emptyset \text {, since relint } \mathbb{Q}(\{\mathbf{0}\})=\{\mathbf{0}\} \text {. }
$$

Thus $\mathcal{D}_{\mathcal{O}}^{\mathcal{G}}$ is a simplex, so $\mathcal{D}_{\mathcal{G}}^{\mathcal{G}}$ and $\mathcal{D}_{\mathcal{F}}^{\mathcal{G}}$ are subcomplexes of $\mathcal{D}_{\mathcal{O}}^{\mathcal{G}}$. 
To every polynomial in $K\left[x_{1}, \ldots, x_{n}\right]$ we are going to assign a series of simplicial complexes, one for each group $\mathcal{G}$ and a specialization $\mathcal{F}$ of $\mathcal{G}$. Recall that $A=\left\{\mathbf{a}_{1}, \ldots, \mathbf{a}_{n}\right\}$ is a set of vectors such that $\operatorname{Sat}\left(L_{\mathcal{G}}\right)=L_{\mathbb{Z} A}$. Let $N=x_{i_{1}}^{n_{1}} \cdots x_{i_{s}}^{n_{s}}$ be a monomial in $K\left[x_{1}, \ldots, x_{n}\right]$. Set $A_{N}:=\left\{\mathbf{a}_{i_{1}}, \ldots, \mathbf{a}_{i_{s}}\right\}$, the cone of $N$ is

$$
\operatorname{cone}(N):=\bigcap_{A_{N} \subset \sigma_{\mathcal{G}}(E)} \sigma_{\mathcal{G}}(E) \subset \sigma_{\mathcal{G}}
$$

Let $F$ be a polynomial in $K\left[x_{1}, \ldots, x_{n}\right]$. We associate with $F$ the induced subcomplex $\mathcal{D}_{\mathcal{G}}^{\mathcal{G}}(F)$ of $\mathcal{D}_{\mathcal{G}}^{\mathcal{G}}$ consisting of those vertices $\mathbb{E}_{i}$ with the property: there exist a monomial $N$ in $F$ such that $\operatorname{cone}(N)=\sigma_{\mathcal{G}}\left(\mathbb{E}_{i}\right)$. Let $\mathcal{D}_{\mathcal{F}}^{\mathcal{G}}(F)$ be the subcomplex of $\mathcal{D}_{\mathcal{F}}^{\mathcal{G}}$ induced on the vertices of $\mathcal{D}_{\mathcal{G}}^{\mathcal{G}}(F)$.

Theorem 3.7. Let $\mathcal{F} \leq \mathcal{G}$ be finitely generated abelian groups. If $F_{1}, \ldots, F_{s}$ generate $\operatorname{rad}\left(I_{L_{\mathcal{G}}}\right)$ up to radical, then $\bigcup_{i=1}^{s} \mathcal{D}_{\mathcal{F}}^{\mathcal{G}}\left(F_{i}\right)$ is a spanning subcomplex of $\mathcal{D}_{\mathcal{F}}^{\mathcal{G}}$.

Proof. Let $\mathbb{E}_{i}$ be a vertex of $\mathcal{D}_{\mathcal{F}}^{\mathcal{G}}$. Then $\mathbb{E}_{i}$ is a vertex of $\mathcal{D}_{\mathcal{G}}^{\mathcal{G}}$ and therefore, from Theorem 5.1 in [9], there exists a monomial $N$ in some $F_{j}$ such that $\operatorname{cone}(N)=\sigma_{\mathcal{G}}\left(\mathbb{E}_{i}\right)$. Thus $\bigcup_{i=1}^{s} \mathcal{D}_{\mathcal{F}}^{\mathcal{G}}\left(F_{i}\right)$ is a spanning subcomplex of $\mathcal{D} \mathcal{\mathcal { F }}$.

Proposition 3.8. Let $\mathcal{F} \leq \mathcal{G}$ be finitely generated abelian groups with $n$ generators and let $F \in K\left[x_{1}, \ldots, x_{n}\right]$ be an $\mathcal{F}$-homogeneous polynomial. Then the simplicial complex $\mathcal{D}_{\mathcal{F}}^{\mathcal{G}}(F)$ is a simplex.

Proof. The empty space is a simplex, so it is enough to consider the case where $\mathcal{D}_{\mathcal{F}}^{\mathcal{G}}(F)$ is not empty. Let $A, B$ be two sets of vectors such that $\operatorname{Sat}\left(L_{\mathcal{G}}\right)=L_{\mathbb{Z} A}, \operatorname{Sat}\left(L_{\mathcal{F}}\right)=L_{\mathbb{Z} B}$ and let $T$ be the set of vertices of $\mathcal{D}_{\mathcal{F}}^{\mathcal{G}}(F)$. Then for every $\mathbb{E}_{i} \in T$ there exists a monomial $N_{i}=\mathbf{x}^{\mathbf{u}_{i}}$ in $F$ such that $\operatorname{deg}_{\mathbb{Z} A}\left(\mathbf{u}_{i}\right) \in$ relint $_{\mathbb{Q}}\left(\sigma_{\mathcal{G}}\left(\mathbb{E}_{i}\right)\right)$, see the proof of Theorem 5.1 in [9. Consequently $\pi\left(\operatorname{deg}_{\mathbb{Z} A}\left(\mathbf{u}_{i}\right)\right)$ belongs to relint $\mathbb{Q}_{\mathbb{Q}}\left(\pi\left(\sigma_{\mathcal{G}}\left(\mathbb{E}_{i}\right)\right)\right)$ and therefore $\operatorname{deg}_{\mathbb{Z} B}\left(\mathbf{u}_{i}\right) \in$ relint $_{\mathbb{Q}}\left(\pi\left(\sigma_{\mathcal{G}}\left(\mathbb{E}_{i}\right)\right)\right)$ since $\operatorname{deg}_{\mathbb{Z} B}\left(\mathbf{u}_{i}\right)=\pi\left(\operatorname{deg}_{\mathbb{Z} A}\left(\mathbf{u}_{i}\right)\right)$. But $F$ is $\mathbb{Z} B$-homogeneous, so $\operatorname{deg}_{\mathbb{Z} B}\left(\mathbf{u}_{i}\right)$ is the same for all monomials in $F$. Hence

$$
\operatorname{deg}_{\mathbb{Z} B}\left(\mathbf{u}_{i}\right) \in \bigcap_{\mathbb{E}_{i} \in T} \text { relint }_{\mathbb{Q}}\left(\pi\left(\sigma_{\mathcal{G}}\left(\mathbb{E}_{i}\right)\right)\right) .
$$

Thus

$$
\bigcap_{\mathbb{E}_{i} \in T} \operatorname{relint}_{\mathbb{Q}}\left(\pi\left(\sigma_{\mathcal{G}}\left(\mathbb{E}_{i}\right)\right)\right) \neq \emptyset
$$

which implies that $T \in \mathcal{D}_{\mathcal{F}}^{\mathcal{G}}$ and then also $T \in \mathcal{D}_{\mathcal{F}}^{\mathcal{G}}(F)$, since $\mathcal{D}_{\mathcal{F}}^{\mathcal{G}}(F)$ is an induced subcomplex. Consequently $\mathcal{D}_{\mathcal{F}}^{\mathcal{G}}(F)$ is a simplex. lary:

Combining Theorem 3.7 with Proposition 3.8 we get the following corol-

Corollary 3.9. Let $\mathcal{F} \leq \mathcal{G}$ be finitely generated abelian groups with $n$ generators. If $F_{1}, \ldots, F_{s}$ are $\mathcal{F}$-homogeneous polynomials and generate $\operatorname{rad}\left(I_{L_{\mathcal{G}}}\right)$ up to radical, then $\bigcup_{i=1}^{s} \mathcal{D}_{\mathcal{F}}^{\mathcal{G}}\left(F_{i}\right)$ is a spanning subcomplex of $\mathcal{D}_{\mathcal{F}}^{\mathcal{G}}$ and each $\mathcal{D}_{\mathcal{F}}^{\mathcal{G}}\left(F_{i}\right)$ is a simplex. 
We can use Corollary 3.9 to provide a lower bound for $\operatorname{ara}_{\mathbb{Z} B}\left(I_{L_{\mathcal{G}}}\right)$, where $B$ is a set of vectors such that $\operatorname{Sat}\left(L_{\mathcal{F}}\right)=L_{\mathbb{Z} B}$.

Let $\mathcal{D}$ be a simplicial complex with vertices $\mathcal{V}=\left\{v_{1}, \ldots, v_{n}\right\}$ and $\Omega=$ $\{0,1, \ldots, \operatorname{dim}(\mathcal{D})\}$. A set $\mathcal{M}=\left\{T_{1}, \ldots, T_{s}\right\}$ of simplices of $\mathcal{D}$ is called an $\Omega$-matching in $\mathcal{D}$ if $T_{k} \cap T_{l}=\emptyset$, for all distinct indices $k$ and $l$, see also Definition 2.1 in [10]. Let $\operatorname{supp}(\mathcal{M})=\cup_{i=1}^{s} T_{i}$, which is a subset of the set of vertices $\mathcal{V}$. We denote by $\operatorname{card}(\mathcal{M})$ the cardinality $s$ of the set $\mathcal{M}$. An $\Omega$-matching $\mathcal{M}$ in $\mathcal{D}$ is called a maximal $\Omega$-matching if $\sup p(\mathcal{M})$ has the maximum possible cardinality among all $\Omega$-matchings. By $\delta(\mathcal{D})_{\Omega}$ we denote the minimum $\operatorname{card}(\mathcal{M})$ among all maximal $\Omega$-matchings $\mathcal{M}$ in $\mathcal{D}$. For a simplicial complex $\mathcal{D}$ the number $\delta(\mathcal{D})_{\Omega}$ is equal to the smallest number $s$ of simplices $T_{i}$ of $\mathcal{D}$ such that the subcomplex $\cup_{i=1}^{s} T_{i}$ is spanning, see Proposition 3.3 in [10]. These numbers were introduced in [10], where we proved that $\delta\left(\mathcal{D}_{\mathcal{G}}^{\mathcal{G}}\right)_{\Omega} \leq \operatorname{ara}_{\mathcal{G}}\left(I_{L_{\mathcal{G}}}\right)$. To every simplicial complex $\mathcal{D}$ we can associate a simple graph, called the $\{0,1\}$-skeleton of $\mathcal{D}$ and denoted by $\mathbb{G}(\mathcal{D})$, formed by the simplices of $\mathcal{D}$ of dimension at most 1 . The complement of $\mathbb{G}(\mathcal{D})$, denoted by $\overline{\mathbb{G}(\mathcal{D})}$, is the graph with the same vertices as $\mathbb{G}(\mathcal{D})$, such that there is an edge between the vertices $v_{i}$ and $v_{j}$ if and only if there is no edge between $v_{i}$ and $v_{j}$ in the graph $\mathbb{G}(\mathcal{D})$. Given an integer $k$, a $k$-coloring of $\overline{\mathbb{G}(\mathcal{D})}$ is a function $c: \mathcal{V} \rightarrow\{1, \ldots, k\}$ such that $c\left(v_{i}\right) \neq c\left(v_{j}\right)$ if the vertices $v_{i}, v_{j}$ are joined by an edge of $\overline{\mathbb{G}(\mathcal{D})}$. The chromatic number $\gamma(\overline{\mathbb{G}(\mathcal{D})})$ of $\overline{\mathbb{G}(\mathcal{D})}$ is the smallest integer $k$ such that there is a $k$-coloring of $\overline{\mathbb{G}(\mathcal{D})}$.

Combining Corollary 3.9 with Corollary 2.12 in [10] we have the following Theorem:

Theorem 3.10. Let $\mathcal{F} \leq \mathcal{G}$ be finitely generated abelian groups with $n$ generators and $B$ a set of vectors such that $\operatorname{Sat}\left(L_{\mathcal{F}}\right)=L_{\mathbb{Z} B}$, then

$$
\gamma\left(\overline{\left(\mathbb{G}\left(\mathcal{D}_{\mathcal{F}}^{\mathcal{G}}\right)\right.}\right) \leq \delta\left(\mathcal{D}_{\mathcal{F}}^{\mathcal{G}}\right)_{\Omega} \leq \operatorname{ara}_{\mathbb{Z} B}\left(I_{L_{\mathcal{G}}}\right) \leq \operatorname{ara}_{\mathcal{F}}\left(I_{L_{\mathcal{G}}}\right) .
$$

In the case that the finest $\mathcal{H}$-grading of Theorem 3.4 is given by the zero group the lower bound given by Theorem 3.10 does not provide actually any information about the arithmetical rank of a lattice ideal. Even in this case the next theorem provides information about the size and the complexity of the polynomials $F_{1}, \ldots, F_{s}$ which generate $\operatorname{rad}\left(I_{L_{\mathcal{G}}}\right)$ up to radical.

Theorem 3.11. Let $I_{L_{\mathcal{G}}}$ be a lattice ideal and $\mathcal{F}$ a specialization of $\mathcal{G}$. If $F_{1}, \ldots, F_{s}$ generate $\operatorname{rad}\left(I_{L_{\mathcal{G}}}\right)$ up to radical, then

(1) the total number of monomials in the nonzero terms of the polynomials $F_{1}, \ldots, F_{s}$ is greater than or equal to the number of vertices of $\mathcal{D}_{\mathcal{G}}^{\mathcal{G}}$ and

(2) the total number of $\mathcal{F}$-homogeneous components in $F_{1}, \ldots, F_{s}$ is greater than or equal to $\delta\left(\mathcal{D}_{\mathcal{F}}^{\mathcal{G}}\right)_{\Omega}$.

Proof. (1) Using Theorem 3.7 we take that for each vertex $\mathbb{E}_{i}$ of $\mathcal{D}_{\mathcal{G}}^{\mathcal{G}}$ there exists at least one monomial $N$ in a nonzero term of some $F_{j}$, such that cone $(N)=\sigma_{\mathcal{G}}\left(\mathbb{E}_{i}\right)$. The result follows.

(2) Let $F_{i}\left(\mathbf{b}_{i, 1}\right), \ldots, F_{i}\left(\mathbf{b}_{i, q_{i}}\right)$ be all the $\mathcal{F}$-homogeneous components of $F_{i}$, 
$1 \leq i \leq s$. Then

$$
\operatorname{rad}\left(I_{L_{\mathcal{G}}}\right)=\operatorname{rad}\left(F_{1}\left(\mathbf{b}_{1,1}\right), \ldots, F_{1}\left(\mathbf{b}_{1, q_{1}}\right), \ldots, F_{s}\left(\mathbf{b}_{s, 1}\right), \ldots, F_{s}\left(\mathbf{b}_{s, q_{s}}\right)\right)
$$

since

$$
\left(F_{1}, \ldots, F_{s}\right) \subset\left(F_{1}\left(\mathbf{b}_{1,1}\right), \ldots, F_{1}\left(\mathbf{b}_{1, q_{1}}\right), \ldots, F_{s}\left(\mathbf{b}_{s, 1}\right), \ldots, F_{s}\left(\mathbf{b}_{s, q_{s}}\right)\right) \subset I_{L_{\mathcal{G}}} .
$$

Thus $\operatorname{ara}_{\mathcal{F}}\left(I_{L_{\mathcal{G}}}\right) \leq q_{1}+\cdots+q_{s}$ and therefore, from Theorem 3.10, we have that $\delta\left(\mathcal{D}_{\mathcal{F}}^{\mathcal{G}}\right)_{\Omega} \leq q_{1}+\cdots+q_{s}$.

\section{Application}

In this section we will give an example of a toric ideal $I_{L_{\mathbb{Z} A_{G}}}$ to explain how the techniques of the previous sections can be applied to give lower bounds for the $\mathcal{F}$-homogeneous arithmetical rank. For the toric ideal $I_{L_{\mathbb{Z} A_{G}}}$ we prove that:

(1) it is not a $\mathbb{Z} B$-homogeneous, as well as $\mathbb{Z} A_{G}$-homogeneous, settheoretic complete intersection, for a certain specialization $\mathbb{Z} B$ of $\mathbb{Z} A_{G}$.

(2) it is not an $\mathcal{F}$-homogeneous set-theoretic complete intersection, for infinitely many specializations $\mathcal{F}$ of $\mathbb{Z} A_{G}$.

One can use the techniques, based on circuits of a vector configuration, developed in [10] to compute the simplicial complex $\mathcal{D}_{\mathcal{G}}^{\mathcal{G}}$ and therefore find the vertices $\left\{\mathbb{E}_{1}, \ldots, \mathbb{E}_{f}\right\}$ of the simplicial complex $\mathcal{D}_{\mathcal{F}}^{\mathcal{G}}$. Explicitly computing the intersections of the relative interiors of the cones $\pi\left(\sigma_{\mathcal{G}}\left(\mathbb{E}_{i}\right)\right)$ we obtain the simplices of $\mathcal{D}_{\mathcal{F}}^{\mathcal{G}}$. Using all these informations we can compute the chromatic number of the complement of the $\{0,1\}$-skeleton of $\mathcal{D} \mathcal{G}$, which provides a lower bound for the $\mathcal{F}$-homogeneous arithmetical rank.

Let $G$ be the graph cube

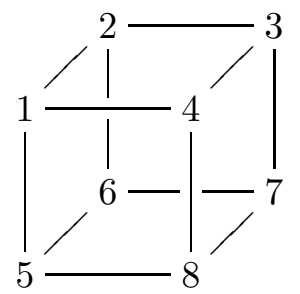

To every graph we can assign a toric ideal in the polynomial ring with so many variables as the edges of the graph. This toric ideal is commonly known as the toric ideal arising from the graph $G$. More details about toric ideals arising from finite graphs can be found in [17] and in [15]. Let $A_{G}$ be the set of all vectors $\mathbf{a}_{i j}=\mathbf{e}_{i}+\mathbf{e}_{j}$ such that $\left\{t_{i}, t_{j}\right\}, i<j$, is an edge of $G$, where $\left\{\mathbf{e}_{i} \mid 1 \leq i \leq 8\right\}$ is the canonical basis of $\mathbb{R}^{8}$. Note that every vector configuration coming from a graph is extremal. A vector configuration $A$ is called extremal if the strongly convex rational polyhedral cone $\sigma_{\mathbb{Z} A}$ is not generated by any proper subset of $A$. Let $\mathcal{F}=\mathbb{Z} B$ and $\mathcal{G}=\mathbb{Z} A_{G}$. Consider the toric ideal

$$
I_{L_{\mathcal{G}}} \subset K\left[x_{12}, x_{14}, x_{15}, x_{23}, x_{26}, x_{34}, x_{37}, x_{48}, x_{56}, x_{58}, x_{67}, x_{78}\right] .
$$


It is the kernel of the $K$-algebra homomorphism

$$
\phi: \mathbb{k}\left[x_{12}, x_{14}, x_{15}, x_{23}, x_{26}, x_{34}, x_{37}, x_{48}, x_{56}, x_{58}, x_{67}, x_{78}\right] \rightarrow \mathbb{k}\left[t_{1}, \ldots, t_{8}\right]
$$

defined by $\phi\left(x_{i j}\right)=\mathbf{t}^{\mathbf{a}_{i j}}$. There are 6 circuits corresponding to the cycles of length 4, 16 circuits corresponding to the cycles of length 6 and 6 circuits corresponding to the cycles of length 8 . More precisely the circuits of $A_{G}$ are

$$
\begin{aligned}
\mathcal{C}_{A_{G}}= & \left\{x_{14} x_{23}-x_{12} x_{34}, x_{12} x_{56}-x_{15} x_{26}, x_{26} x_{37}-x_{23} x_{67}, x_{14} x_{58}-x_{15} x_{48},\right. \\
& x_{37} x_{48}-x_{34} x_{78}, x_{58} x_{67}-x_{56} x_{78}, x_{23} x_{48} x_{56}-x_{26} x_{34} x_{58}, \\
& x_{14} x_{37} x_{56}-x_{15} x_{34} x_{67}, x_{12} x_{37} x_{58}-x_{15} x_{23} x_{78}, x_{12} x_{48} x_{67}-x_{14} x_{26} x_{78}, \\
& x_{23} x_{56} x_{78}-x_{26} x_{37} x_{58}, x_{14} x_{56} x_{78}-x_{15} x_{48} x_{67}, x_{26} x_{34} x_{78}-x_{23} x_{48} x_{67}, \\
& x_{15} x_{34} x_{78}-x_{14} x_{37} x_{58}, x_{15} x_{26} x_{78}-x_{12} x_{58} x_{67}, x_{14} x_{23} x_{78}-x_{12} x_{37} x_{48}, \\
& x_{34} x_{58} x_{67}-x_{37} x_{48} x_{56}, x_{12} x_{34} x_{67}-x_{14} x_{26} x_{37}, x_{15} x_{23} x_{67}-x_{12} x_{37} x_{56}, \\
& x_{12} x_{34} x_{58}-x_{15} x_{23} x_{48}, x_{14} x_{26} x_{58}-x_{12} x_{48} x_{56}, x_{14} x_{23} x_{56}-x_{15} x_{26} x_{34}, \\
& x_{12} x_{34} x_{56} x_{78}-x_{15} x_{23} x_{48} x_{67}, x_{12} x_{34} x_{56} x_{78}-x_{14} x_{26} x_{37} x_{58}, \\
& x_{14} x_{23} x_{56} x_{78}-x_{15} x_{26} x_{37} x_{48}, x_{12} x_{34} x_{58} x_{67}-x_{15} x_{26} x_{37} x_{48}, \\
& \left.x_{14} x_{23} x_{58} x_{67}-x_{12} x_{37} x_{48} x_{56}, x_{14} x_{23} x_{58} x_{67}-x_{15} x_{26} x_{34} x_{78}\right\} .
\end{aligned}
$$

Looking at the monomials of the above circuits and considering their minimal elements, which are the 20 monomials involved in the first ten circuits, we get all the vertices of the simplicial complex $\mathcal{D}_{\mathcal{G}}^{\mathcal{G}}$, see Section 4 [10. The complex $\mathcal{D}_{\mathcal{G}}^{\mathcal{G}}$ has 20 vertices defined by the following sets:

$\mathbb{E}_{1}=\{14,23\}, \mathbb{E}_{2}=\{12,34\}, \mathbb{E}_{3}=\{12,56\}, \mathbb{E}_{4}=\{15,26\}, \mathbb{E}_{5}=\{26,37\}$,

$\mathbb{E}_{6}=\{23,67\}, \mathbb{E}_{7}=\{14,58\}, \mathbb{E}_{8}=\{15,48\}, \mathbb{E}_{9}=\{37,48\}, \mathbb{E}_{10}=\{34,78\}$,

$\mathbb{E}_{11}=\{58,67\}, \mathbb{E}_{12}=\{56,78\}, \mathbb{E}_{13}=\{23,48,56\}, \mathbb{E}_{14}=\{26,34,58\}$,

$\mathbb{E}_{15}=\{14,37,56\}, \mathbb{E}_{16}=\{15,34,67\}, \mathbb{E}_{17}=\{12,37,58\}, \mathbb{E}_{18}=\{15,23,78\}$,

$\mathbb{E}_{19}=\{12,48,67\}, \mathbb{E}_{20}=\{14,26,78\}$.

It has ten 1-simplices, namely

$$
\begin{gathered}
\left\{\mathbb{E}_{1}, \mathbb{E}_{2}\right\},\left\{\mathbb{E}_{3}, \mathbb{E}_{4}\right\},\left\{\mathbb{E}_{5}, \mathbb{E}_{6}\right\},\left\{\mathbb{E}_{7}, \mathbb{E}_{8}\right\},\left\{\mathbb{E}_{9}, \mathbb{E}_{10}\right\}, \\
\left\{\mathbb{E}_{11}, \mathbb{E}_{12}\right\},\left\{\mathbb{E}_{13}, \mathbb{E}_{14}\right\},\left\{\mathbb{E}_{15}, \mathbb{E}_{16}\right\},\left\{\mathbb{E}_{17}, \mathbb{E}_{18}\right\},\left\{\mathbb{E}_{19}, \mathbb{E}_{20}\right\} .
\end{gathered}
$$

There are no 2 -simplices. The first ten binomials of $\mathcal{C}_{A_{G}}$ constitute a minimal set of generators of the ideal $I_{L_{\mathcal{G}}}$ and therefore $\operatorname{ara\mathcal {G}}\left(I_{L_{\mathcal{G}}}\right) \leq 10$. On the other hand the chromatic number of the complement of the $\{0,1\}$-skeleton of $\mathcal{D}_{\mathcal{G}}^{\mathcal{G}}$ is equal to 10, so $\operatorname{araG}_{\mathcal{G}}\left(I_{L_{\mathcal{G}}}\right)=10$, see Section 4 in [10].

Consider the set of vectors

$$
\begin{aligned}
B= & \left\{\mathbf{b}_{12}=(5,0,3,4), \mathbf{b}_{14}=(3,1,5,5), \mathbf{b}_{15}=(4,1,4,8), \mathbf{b}_{23}=(4,0,2,3),\right. \\
& \mathbf{b}_{26}=(5,0,1,6), \mathbf{b}_{34}=(2,1,4,4), \mathbf{b}_{37}=(2,1,2,6), \mathbf{b}_{48}=(1,2,5,8), \\
& \left.\mathbf{b}_{56}=(4,1,2,10), \mathbf{b}_{58}=(2,2,4,11), \mathbf{b}_{67}=(3,1,1,9), \mathbf{b}_{78}=(1,2,3,10)\right\} .
\end{aligned}
$$

We have that $\mathcal{F}$ is a specialization of $\mathcal{G}$, since $L_{\mathcal{G}} \subset L_{\mathcal{F}}$. Let $\pi: \sigma_{\mathcal{G}} \rightarrow \sigma_{\mathcal{F}}$ be the projection of cones, given by $\pi\left(\mathbf{a}_{i j}\right)=\mathbf{b}_{i j}$. We will compute the simplices of $\mathcal{D}_{\mathcal{F}}^{\mathcal{G}}$. The vertices of $\mathcal{D}_{\mathcal{F}}^{\mathcal{G}}$ are the same with $\mathcal{D}_{\mathcal{G}}^{\mathcal{G}}$, namely $\mathbb{E}_{1}, \ldots, \mathbb{E}_{20}$. There are 20 cones of the form $\pi\left(\sigma_{\mathcal{G}}\left(\mathbb{E}_{i}\right)\right)$, i.e.

$$
\pi\left(\sigma_{\mathcal{G}}\left(\mathbb{E}_{1}\right)\right)=\operatorname{pos}_{\mathbb{Q}}\left(\mathbf{b}_{14}, \mathbf{b}_{23}\right), \pi\left(\sigma_{\mathcal{G}}\left(\mathbb{E}_{2}\right)\right)=\operatorname{pos}_{\mathbb{Q}}\left(\mathbf{b}_{12}, \mathbf{b}_{34}\right) \text { etc. }
$$

By explicitly computing the intersections of the relative interiors of the above cones we take that the simplicial complex $\mathcal{D}_{\mathcal{F}}^{\mathcal{G}}$ has 7 facets:

(1) one 7-simplex, namely $\left\{\mathbb{E}_{13}, \mathbb{E}_{14}, \mathbb{E}_{15}, \mathbb{E}_{16}, \mathbb{E}_{17}, \mathbb{E}_{18}, \mathbb{E}_{19}, \mathbb{E}_{20}\right\}$. 
(2) six 1-simplices, namely

$$
\left\{\mathbb{E}_{1}, \mathbb{E}_{2}\right\},\left\{\mathbb{E}_{3}, \mathbb{E}_{4}\right\},\left\{\mathbb{E}_{5}, \mathbb{E}_{6}\right\},\left\{\mathbb{E}_{7}, \mathbb{E}_{8}\right\},\left\{\mathbb{E}_{9}, \mathbb{E}_{10}\right\},\left\{\mathbb{E}_{11}, \mathbb{E}_{12}\right\}
$$

Note that $\Omega=\{0,1, \ldots, 7\}$. We have that $\delta\left(\mathcal{D}_{\mathcal{F}}^{\mathcal{G}}\right)_{\Omega}=7$, attained by the maximal $\Omega$-matching

$\left\{\left\{\mathbb{E}_{1}, \mathbb{E}_{2}\right\},\left\{\mathbb{E}_{3}, \mathbb{E}_{4}\right\},\left\{\mathbb{E}_{5}, \mathbb{E}_{6}\right\},\left\{\mathbb{E}_{7}, \mathbb{E}_{8}\right\},\left\{\mathbb{E}_{9}, \mathbb{E}_{10}\right\},\left\{\mathbb{E}_{11}, \mathbb{E}_{12}\right\},\left\{\mathbb{E}_{13}, \mathbb{E}_{14}, \ldots, \mathbb{E}_{20}\right\}\right\}$.

Remark that $\gamma\left(\overline{\mathbb{G}\left(\mathcal{D}_{\mathcal{F}}^{\mathcal{G}}\right)}\right)=7$. Therefore $7 \leq \operatorname{ara}_{\mathcal{F}}\left(I_{L_{\mathcal{G}}}\right)$. Moreover

$$
\begin{aligned}
\operatorname{rad}\left(I_{L_{\mathcal{G}}}\right)= & \operatorname{rad}\left(x_{14} x_{23}-x_{12} x_{34}, x_{12} x_{56}-x_{15} x_{26}, x_{26} x_{37}-x_{23} x_{67},\right. \\
& x_{14} x_{58}-x_{15} x_{48}, x_{37} x_{48}-x_{34} x_{78}, x_{58} x_{67}-x_{56} x_{78}, \\
& \left(x_{23} x_{48} x_{56}-x_{26} x_{34} x_{58}\right)+\left(x_{14} x_{37} x_{56}-x_{15} x_{34} x_{67}\right)+ \\
& \left.+\left(x_{12} x_{37} x_{58}-x_{15} x_{23} x_{78}\right)+\left(x_{12} x_{48} x_{67}-x_{14} x_{26} x_{78}\right)\right) .
\end{aligned}
$$

So $\operatorname{ara}_{\mathcal{F}}\left(I_{L_{\mathcal{G}}}\right)=7$. Note also that, since the graph $G$ is bipartite, the height of the toric ideal $I_{L_{\mathcal{G}}}$ is equal to the number of edges minus the number of vertices plus one, see [17], so $h t\left(I_{L_{\mathcal{G}}}\right)=5$, which implies that $I_{L_{\mathcal{G}}}$ is not a $\mathcal{F}$-homogeneous set-theoretic complete intersection. Actually for any group $\mathcal{H}$ such that $\mathcal{F} \leq \mathcal{H} \leq \mathcal{G}$ the toric ideal $I_{L_{\mathcal{G}}}$ is not an $\mathcal{H}$-homogeneous set-theoretic complete intersection, since

$$
5 \leq \operatorname{ara\mathcal {F}}\left(I_{L_{\mathcal{G}}}\right)=7 \leq \operatorname{ara\mathcal {H}}\left(I_{L_{\mathcal{G}}}\right) \leq \operatorname{ara\mathcal {G}}\left(I_{L_{\mathcal{G}}}\right)=10 .
$$

There are infinitely many different equivalent classes of $\mathcal{H}$ 's since the rank of $\mathcal{F}$ equals 8 and the rank of $\mathcal{G}$ equals 5 .

\section{Acknowledgment}

The authors thank the referee for his careful reading of the manuscript and his helpful remarks.

\section{REFERENCES}

[1] Barile, M. On a special class of simplicial toric varieties, J. Algebra 308 (2007) 368382.

[2] Barile, M., Lyubeznik, G. Set-theoretic complete intersections in characteristic p, Proc. Amer. Math. Soc. 133 (2005) 3199-3209.

[3] Cox, D. A. The homogeneous coordinate ring of a toric variety, J. Algebraic Geom. 4 (1995) $17-50$

[4] D. Eisenbud, Commutative Algebra with a view toward Algebraic Geometry. Springer Verlag New York, Berlin, Heidelberg 1994.

[5] Eisenbud, D., Mustata, M., Stillman, M. Cohomology of Toric varieties and Local cohomology with monomial supports, J. Symbolic Computation 29 (2000) 583-600.

[6] Eto, K. Set-theoretic complete intersection lattice ideals in monoid rings, J. Algebra 299 (2006) 689-706.

[7] Geiger, D., Meek, C., Sturmfels, B. On the toric algebra of graphical models, Ann. Statist. 34 (2006) 1463-1492.

[8] Katsabekis, A. Projections of cones and the arithmetical rank of toric varieties, J. Pure Appl. Algebra 199 (2005) 133-147.

[9] Katsabekis, A., Morales, M., Thoma, A. Stanley-Reisner rings and the radicals of lattice ideals, J. Pure Appl. Algebra 204 (2006) 584-601.

[10] Katsabekis, A., Thoma, A. Matchings in simplicial complexes, circuits and toric varieties, J. Comb. Theory, Ser. A, 114 (2007) 300-310.

[11] Katsabekis, A., Thoma, A. Parametrizations of toric varieties over any field, J. Algebra 308 (2007) 751-763.

[12] Knutson, A., Miller, E. Gröbner geometry of Schubert polynomials, Ann. Math 161 (2005) 1245-1318. 
[13] Kreuzer, M., Robbiano, L. Computational Commutative Algebra 2, Springer-Verlag, Berlin, 2005.

[14] Miller, E., Sturmfels, B. Combinatorial Commutative Algebra. Graduate Texts in Mathematics 227, Springer-Verlag New York, 2005.

[15] Ohsugi, H., Hibi, T. Toric ideals generated by quadratic binomials, J. Algebra 218 (1999) 509-527.

[16] B. Sturmfels, Gröbner Bases and Convex Polytopes. University Lecture Series, No. 8 American Mathematical Society Providence, R.I. 1995.

[17] Villarreal, R. H. Rees algebras of edge ideals, Comm. Algebra 23 (1995) 3513-3524.

Department of Mathematics, University of the Aegean, 83200 Karlovassi, SAMOS, GREECE

E-mail address: katsabek@aegean.gr

Department of Mathematics, University of IoAnnina, IoAnnina 45110, GREeCE

E-mail address: athoma@uoi.gr 